\title{
Pleural Hemorrhage, CTCAE
}

National Cancer Institute

\section{Source}

National Cancer Institute. Pleural Hemorrhage, CTCAE. NCI Thesaurus. Code C56558.

A disorder characterized by bleeding from the pleural cavity. 